\title{
The versatile RECQL4
}

\author{
Richard Kellermayer, $M D, P h D$
}

\begin{abstract}
The human DNA helicase RECQL4 interacts in an array of intracellular regulatory pathways from the initiation of DNA replication, through maintaining genomic stability, to the N-end rule pathway. Interestingly, mutations in RECQL4 have recently been revealed not only in Rothmund-Thomson-, but RAPADILINO-, and cases of Baller-Gerold syndrome also. Although these disorders represent distinct genetic entities, clinical observations have delineated highly variable expressivity and significant overlaps in the associated phenotypic manifestations. Consequently, it is especially difficult to draw precise genotype-phenotype correlations in RECQL4 related syndromes. This is likely due to the complex and multiple cellular networks RECQL4 is associated with. Genet Med 2006:8(4):213-216.
\end{abstract}

Key Words: RECQL4, Rothmund-Thomson syndrome, RAPADILINO, Baller-Gerold syndrome, helicase

\section{INTRODUCTION}

Helicases are a highly conserved group of enzymes that unwind RNA or DNA in an ATP-dependent manner. These proteins function in processes where access to single-stranded nucleic acid is required, including DNA replication, DNA repair and recombination, and transcription of RNA. ${ }^{1,2}$ Humans and other mammals contain at least five different helicases of the RecQ family, named after the single RecQ gene of Escherichia coli. ${ }^{3}$ Deficiencies of these proteins have been associated with genomic instability disorders of man with several features of premature aging and/or cancer predisposition..$^{4-6}$ Mutations in the RECQ-like genes BLM, WRN, and RECQL4 can result in Bloom syndrome (MIM \#210900), Werner syndrome (MIM \#277700), and Rothmund-Thomson syndrome/RTS/(MIM \#268400), respectively. ${ }^{4}$ However, RECQL4 stands unique among these genes in several respects, among which the most apparent to a medical geneticist is the fact that it has been related not only to RTS, but to RAPADILINO syndrome (MIM \#266280), and to Baller-Gerold syndrome (MIM \#218600) also. ${ }^{7,8}$ Interestingly, significant overlaps may be present in the phenotypic manifestations of these RECQL4-related genetic disorders and phenotype-genotype correlations are hard to delineate. ${ }^{8}$ This review intends to highlight the recent advancements in the molecular biology of RECQL4 in relationship to the observed significant phenotypic variability of the associated genetic syndromes.

\section{RECQL4}

The RECQL4 gene in located on human chromosome $8 \mathrm{q} 24.3$, containing 21 exons in less than $6.5 \mathrm{~kb}$ of genomic

\footnotetext{
From the Department of Medical Genetics and Child Development, University of Pécs, Hungary. Richard Kellermayer, MD, PhD, University of Pécs, Department of Medical Genetics, József A. u. 7., 7623 Pécs, Hungary

Submitted for publication October 27, 2005.

Accepted for publication January 9, 2006.
}

DOI: 10.1097/01.gim.0000214457.58378.1a sequence, with 13 introns that are $<100$ bp in length. ${ }^{9}$ This peculiar gene structure predisposes mutated RECQL4 mRNA to mis-splicing alterations resulting from the mutation in the primary gene transcript. ${ }^{10}$ Consequently, intronic size constraint and point mutations in the introns of the RECQL4 gene have been shown to play a role in the development of RTS in addition to exonic defects. ${ }^{10-12}$

Human RECQL4 encodes a $133 \mathrm{kDa}$ protein of 1,208 amino acids that contains characteristic sequences of the RecQ-family's helicase domain. Namely, a central, highly conserved domain is present in these proteins that consists of 380 amino acids and includes a Walker A-box motif responsible for ATP hydrolysis. However, in contrast to the BLM and WRN helicases, the two C-terminal signature motifs characteristic for the RecQ family are absent from RECQL4. Similarly, the Nuclear Localization Signal residing near the C-terminus of BLM and WRN proteins has not yet been identified in RECQL4. On the other hand, the N-terminal region of RECQL4 uniquely bears homology to the yeast proteins Sld2/DRC1 that are essential for the establishment of replication forks. Yet, RECQL4 has not been shown thus far to actually possess an RNA- or DNA helicase activity. ${ }^{13-16}$ At the cellular level, defects in RECQL4 cause genome instability, particularly trisomy, aneuoploidy and a high frequency of chromosomal rearrangements. ${ }^{17}$ Additionally, somatic cells isolated from RTS individuals are sensitive to ionizing radiation. ${ }^{18}$ Despite this knowledge, the molecular function of RECQL4 and the possible cellular pathways in which it is involved remained poorly understood until recently.

Last year, molecular studies have revealed that at least in HeLa cells, a proportion of RECQL4 forms a stable complex with components of $\mathrm{N}$-end rule pathway ubiquitin ligases, UBR1 and UBR2 in the cytosol. ${ }^{19}$ This pathway has been shown to be essential for chromosome stability in yeast through affecting the degradation of a cohesin sub-unit, that mediates the conditional association of sister chromatids in mitosis and meiosis. ${ }^{20}$ Yet, the precise role of "cytosolic" RECQL4 remains to be elucidated. 
Studies on the nuclear localization of RECQL4 have demonstrated it to be localized in distinct nuclear foci that coincide with proteins involved in the maintenance of genome stability. Namely, RECQL4 foci coincide with those formed by pro-myelocytic leukemia (PML) bodies and RECQL4 forms a complex and co-localizes with Rad51 and regions of single-stranded DNA (ssDNA) upon induction of DNA double-strand breaks (DSBs). ${ }^{21}$ PML body and Rad51 foci have been shown to regulate the response to, and repair of DSBs. ${ }^{22,23}$ These observations suggest that RECQL4 plays a role in the repair of DSBs by homologous recombination. ${ }^{21}$

Xenopus laevis complementation studies have shown that RECQL4 likely plays a role in DNA replication initiation by also promoting the loading of replication factors at initiation sites. ${ }^{13}$

These observations clearly implicate RECQL 4 to function in several aspects of genomic stability maintenance and suggest that it plays an important role in fine-tuning such complex processes as DNA replication initiation and double-strand break repair.

It has been earlier suggested that the expression of RECQL4 is tissue-specific, being most prominent in the thymus and testis. ${ }^{24}$ Others have failed to detect such differences in human tissues, but found that RECQL4 expression is most prominent in the chondrocytes of developing bones and cartilage, as well as in immature, proliferating intestinal enterocytes of embryonic mice older than 15 days post-conception. ${ }^{7}$ RECQL4-deficient mice develop growth retardation and skin abnormalities (i.e., hair loss, colorless hair, brittle skin) in addition to bone dysplasias, intestinal villus atrophy, dental dystrophy and premature aging (death within two weeks). ${ }^{25}$ These results indicate that RECQL4 expression is tissue-specific, especially during embryonic and early postnatal development and that this expression pattern likely accounts for the phenotypic manifestations of genetic syndromes resulting from RECQL4 deficiency.

\section{Rothmund-Thomson syndrome}

Auguste Rothmund and Sydney Thomson described two separate medical conditions which were thought to be part of the same entity and consequently designated Rothmund-Thomson Syndrome (RTS) by William Taylor (MIM \#268400). ${ }^{26-28}$ This is a rare, autosomal recessive disorder characterized by a poikilodermatous rash starting in infancy, cataracts, growth retardation, skeletal abnormalities (i.e., dysplasias, absent or malformed bones [i.e., absent radii], osteopenia, and delayed bone formation), sparse hair and eyebrows, dental anomalies, gastrointestinal disturbances, as well as a high incidence of malignancies, especially osteosarcomas. ${ }^{18,29,30}$ Indeed, osteosarcoma develops in approximately $30 \%$ of the patients with a median age of 11.5 years. ${ }^{30}$ RTS patients have also been reported to rarely develop cutaneous squamous cell carcinoma, non-cutaneous malignancies and myelodysplasia. ${ }^{31,32}$ Regardless of other anomalies, the clinical diagnosis of RTS rests on the manifestation of the poikilodermatous rash. The skin is usually normal at birth and the rash of RTS develops between age three and six months as erythema, swelling, and blistering on the face spreading to the buttocks and extremities. The rash evolves over months to years into the chronic pattern of reticulated hypo- and hyperpigmentation, punctate atrophy, and telangiectases, collectively known as poikiloderma. Even patients with atypical rash or onset have been considered as RTS cases if they have additional features such as radial ray defect, loss of hair, cataracts, osteosarcoma or skeletal dysplasia (Fig. 1). ${ }^{30}$ In 1999, Kitao et al. linked a subset of RTS cases to mutations in the human helicase RECQL4. ${ }^{33}$ As earlier emphasized, observations in RECQL4-deficient mice supported the pathogenic role for this molecular defect in RTS. ${ }^{25}$ A cohort study of 33 patients showed that in $60 \%$ of RTS cases, mutations of both RECQL4 alleles can be found, and the overall RECQL4 mutation detection rate was estimated to be $66 \%$ in RTS. Only one allele was found to carry a mis-sense or a deleterious mutation in another $30 \%$ of the individuals with this disorder. Additionally, linkage studies were negative in one family supporting genetic heterogeneity in this disorder and leaving the possibility of other RTS gene loci open. ${ }^{29}$ Nevertheless, RECQL4 analysis in the same cohort has revealed that all patients with RTS (and without cataracts) who developed osteosarcoma carried at least one deleterious (non-sense or frameshift) RECQL4 mutation. Consequently, it was proposed that two types of RTS exist. Type I associated with poikiloderma and cataracts, but not with osteosarcoma (the form of RTS described by Rothmund); and Type II associated with osteosarcoma and deleterious mutations in RECQL4. ${ }^{14,29}$ However, at least one RTS patient has been reported earlier in whom both juvenile cataracts and osteosarcoma developed, but unfortunately, RECQL4 analysis for the proband was not described..$^{30}$ Nevertheless, these find-

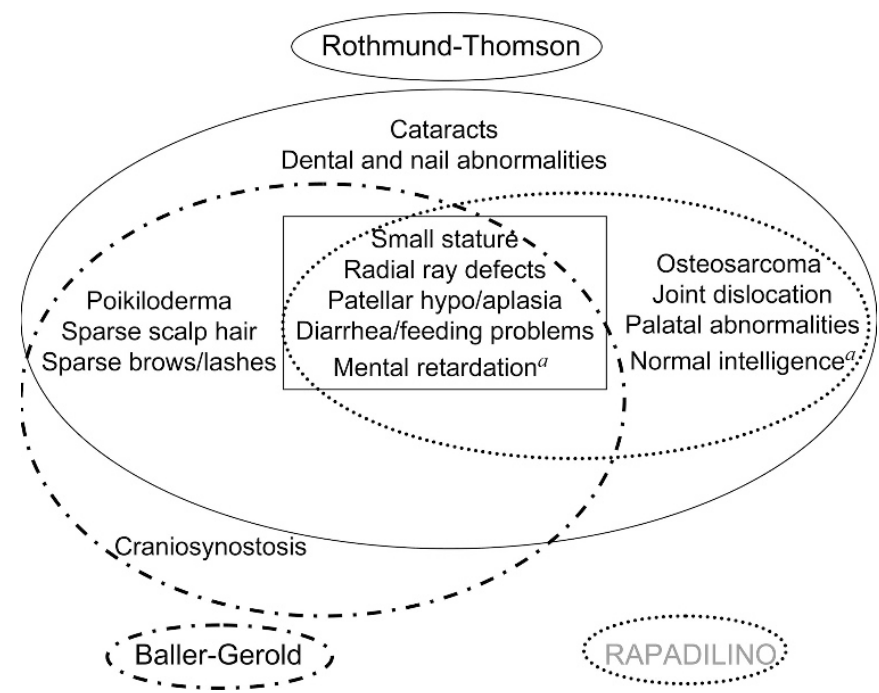

Fig. 1 The phenotypic spectrum of RECQL4 related genetic disorders. The clinical signs specific to each syndrome are circled (RTS: continuous line; BGS: dashed line; RAPADILINO: dotted line). The shared signs of the syndromes are framed in the center of the figure. ${ }^{a}$ Mental retardation has only been incidentally noted in RTS and RAPADILINO, whereas affected patients usually have normal intelligence. While all features of RAPADILINO can be present in RTS, the specific absence of ectodermal symptoms and cataracts delineates RAPADILINO from RTS. The presence of craniosynostosis and the absence of cataracts appear to be the specific features of BGS..$^{8,14,37}$ 
ings indicate that RECQL4 analysis in RTS cases is warranted, and delineating genotype-phenotype correlations is of major importance in respect of prognosis and screening for osteosarcoma. Yet, such correlations have to be handled cautiously due to limited specificity.

\section{RAPADILINO syndrome}

A recent study described RECQL4 mutations in patients with RAPADILINO syndrome (MIM \#266280) also. ${ }^{7}$ The acronym stands for the specific features of this rare autosomal recessive disorder: RAdial hypo-/aplasia, PAtellar hypo-/aplasia, cleft or highly arched PAlate, DIarrhea and DIslocated joints, LIttle size ( $<-2 \mathrm{SD}$ in height) and LImb malformation, slender NOse and NOrmal intelligence. ${ }^{34}$ This entity is most prevalent in Finland (with fourteen patients described), where in all of the observed cases, a specific splice site mutation of the RECQL4 intron 7 (IVS7 + 2delT) was found in either homozygous or compound heterozygous form. This defect possibly leaves the helicase domain of the polypeptide intact and might only partially affect RECQL4 function. The preserved helicase activity may possibly lead to the phenotypic differences and to the lower tumor prevalence when comparing to the RTS patients. ${ }^{7}$ Although several hallmarks of RAPADILINO have been described in RTS cases, their collective appearance and the peculiar absence of ectodermal symptoms (poikiloderma, sparse scalp hair, sparse brows and lashes) in RAPADILINO syndrome have distinguished it as a separate disorder (Fig. 1.). ${ }^{7}$ Yet, a non-Finnish patient with RAPADILINO syndrome has been described earlier in whom poikiloderma developed, but the case was revisited later and classified as a severe form of RTS or a new syndrome. ${ }^{35,36}$ Indeed, despite the differences, there are significant phenotypic overlaps between RTS and RAPADILINO. This observation was further supported by a recent report where a novel compound heterozygous RECQL4 defect (g.2886delT/g.5435C $>$ T) resulted in the phenotypic appearance of all RAPADILINO features in addition to poikiloderma. ${ }^{37}$

\section{Baller-Gerold syndrome}

The similarly rare autosomal recessive Baller-Gerold syndrome (BGS) (MIM \#218600) was delineated following the description of patients with bilateral radial aplasia and craniosynostosis by Baller and Gerold. ${ }^{38,39}$ Other possible phenotypic manifestations of this nosologic entity are short stature, epicanthal folds, hypertelorism, micrognathia, anus malformations (fistula, anteposition, imperforation) and renal anomalies. However, the hallmark of the disorder is lambdoid and coronal craniosynostosis in association with radial hypoplasia. Consequently, only a limited number of additional patients have been reported since the first descriptions, while several atypical forms of the syndrome have been reassigned to other disorders such as Fanconi anemia and Roberts SC phocomelia. ${ }^{8}$ Even patients with characteristic features of Baller-Gerold syndrome can have mutations in the TWIST gene, which is typically associated with Saethre-Chotzen syndrome. ${ }^{40}$ Additionally, FGFR2 mutations may also mimic
BGS. ${ }^{41}$ Such reports led to a narrowed re-definition of BGS based on the exclusion of cytogenetic and hematopoietic abnormalities, as well as the absence of TWIST and FGFR mutations in patients with craniosynostosis and pre-axial upper limb abnormalities. ${ }^{8}$

The development of poikiloderma in two unrelated patients with Baller-Gerold syndrome has prompted investigators to evaluate for RECQL4 defects in these cases recently. RECQL4 mutations were present in both patients, one of whom interestingly possessed the identical compound heterozygous defect (g.2886delT/g.5435C $>$ T) $)^{8}$ as the earlier reported proband mentioned above, in whom all features of RAPADILINO developed in addition to poikiloderma. It appears currently, that the presence of craniosynostosis is the only selective manifestation that delineates BGS from RTS (Fig. 1.).

\section{DISCUSSION}

Recent observations have expanded the number of clinical syndromes related to RECQL4 mutations. Rothmund-Thomson, RAPADILINO and Baller-Gerold syndromes have all been linked to RECQL4 defects.7,8,33 This fact in itself emphasizes the highly variable expressivity of RECQL4 mutations. Most alterations of RECQL4 found in RTS patients represent non-sense or frameshift mutations, but the exception to this rule is frequent. ${ }^{29,33}$ Consequently, precise phenotype-genotype correlations are hard to delineate in the RECQL4 associated genetic disorders. Observations where identical mutations of RECQL4 resulted in either RTS with all features of RAPADILINO $^{37}$ or Baller-Gerold syndrome ${ }^{8}$ underscore this statement. Interestingly, even significant intra-familial variability was noted in the latter report.

The reason for the highly versatile expressivity may lie in the diverse molecular functioning of RECQL4, in addition to potential redundancy with variation in function of the five RECQ proteins, as well as possible proband specific variability in the expression pattern of RECQL4. The intricate networks of associated proteins (which regulate DNA replication initiation and genome stability ${ }^{13,19,21}$ ) are possibly fine-tuned differentially at the cellular, tissue, organ and organism levels. This flexibility may provide room for the variable manifestation of RECQL4 related defects. In this sense, the delineation of RT-, RAPADILINO-, and Baller-Gerold syndromes could be considered as solely semantics once RECQL4 mutations are identified in the affected patients. Yet, the example of the IVS7 + 2delT mutation in RAPADILINO syndrome may indicate that specific RECQL4 mutations can lead to specific phenotypic manifestations, such as the peculiar absence of poikiloderma. This is supported by the observation that despite of some exceptions, osteosarcoma in RTS is mostly associated with deleterious RECQL4 mutations in a complex heterozygous form..$^{29}$ Consequently, further efforts should be made in drawing specific phenotype-genotype correlations to support the prognostics and tumor screening of patients with RECQL4-related syndromes. 


\section{References}

1. Delagoutte E, von Hippel PH. Helicase mechanisms and the coupling of helicases within macromolecular machines. Part I: Structures and properties of isolated helicases. Q Rev Biophys 2002;35:431-478.

2. Delagoutte E, von Hippel PH. Helicase mechanisms and the coupling of helicases within macromolecular machines. Part II: Integration of helicases into cellular processes. Q Rev Biophys 2003;36:1-69.

3. Bachrati CZ, Hickson ID. RecQ helicases: suppressors of tumorigenesis and premature aging. Biochem J 2003;374:577-606.

4. Mohaghegh P, Hickson ID. Premature aging in RecQ helicase-deficient human syndromes. Int J Biochem Cell Biol 2002;34:1496-1501

5. Mohaghegh P, Hickson ID. DNA helicase deficiencies associated with cancer predisposition and premature ageing disorders. Hum Mol Genet 2001;10:741-746.

6. Soultanas P, Wigley DB. Unwinding the 'Gordian knot' of helicase action. Trends Biochem Sci 2001;26:47-54.

7. Siitonen HA, Kopra O, Kaariainen H, Haravuori $\mathrm{H}$ et al. Molecular defect of RAPADILINO syndrome expands the phenotype spectrum of RECQL diseases. Hum Mol Genet 2003;12:2837-2844.

8. Van Maldergem L, Siitonen HA, Jalkh N, Chouery E et al. Revisiting the craniosynostosis-radial ray hypoplasia association: Baller-Gerold syndrome caused by mutations in RECQL4 gene. J Med Genet 2006;43:148-152.

9. Kitao S, Lindor NM, Shiratori M, Furuichi Y et al. Rothmund-thomson syndrome responsible gene, RECQL4: genomic structure and products. Genomics 1999;61: 268-276.

10. Beghini A, Castorina P, Roversi G, Modiano P et al. RNA processing defects of the helicase gene RECQL4 in a compound heterozygous Rothmund-Thomson patient. Am J Med Genet 2003;120:395-399.

11. Wang LL, Worley K, Gannavarapu A, Chintagumpala MM et al. Intron-size constraint as a mutational mechanism in Rothmund-Thomson syndrome. Am J Hum Genet 2002;71:165-167.

12. Balraj P, Concannon P, Jamal R, Beghini A et al. An unusual mutation in RECQ4 gene leading to Rothmund-Thomson syndrome. Mutat Res 2002;508:99-105.

13. Sangrithi MN, Bernal JA, Madine M, Philpott A et al. Initiation of DNA replication requires the RECQL4 protein mutated in Rothmund-Thomson syndrome. Cell 2005;121:887-898.

14. Larizza L, Magnani I, Roversi G. Rothmund-Thomson syndrome and RECQL4 defect: Splitting and lumping. Cancer Lett 2006;232:107-120.

15. Hickson ID. RecQ helicases: caretakers of the genome. Nat Rev Cancer 2003;3:169178.

16. Opresko PL, Cheng WH, Bohr VA. Junction of RecQ helicase biochemistry and human disease. J Biol Chem 2004;279:18099-18102.

17. Der KV, McGill JJ, Vekemans M, Kopelman HR. Clonal lines of aneuploid cells in Rothmund-Thomson syndrome. Am J Med Genet 1990;37:336-339.

18. Vennos EM, James WD. Rothmund-Thomson syndrome. Dermatol Clin 1995;13: 143-150.

19. Yin J, Kwon YT, Varshavsky A, Wang W. RECQL4, mutated in the RothmundThomson and RAPADILINO syndromes, interacts with ubiquitin ligases UBR1 and UBR2 of the N-end rule pathway. Hum Mol Genet 2004;13:2421-2430.
20. Rao H, Uhlmann F, Nasmyth K, Varshavsky A. Degradation of a cohesin subunit by the N-end rule pathway is essential for chromosome stability. Nature 2001;410:955959.

21. Petkovic M, Dietschy T, Freire R, Jiao R et al. The human Rothmund-Thomson syndrome gene product, RECQL4, localizes to distinct nuclear foci that coincide with proteins involved in the maintenance of genome stability. J Cell Sci 2005;118:4261-4269.

22. Carbone R, Pearson M, Minucci S, Pelicci PG. PML NBs associate with the hMrel1 complex and p53 at sites of irradiation induced DNA damage. Oncogene 2002;21: $1633-1640$.

23. Tarsounas M, Davies AA, West SC. RAD51 localization and activation following DNA damage. Philos Trans R Soc Lond B Biol Sci 2004;359:87-93.

24. Kitao S, Ohsugi I, Ichikawa K, Goto M et al. Cloning of two new human helicase genes of the RecQ family: biological significance of multiple species in higher eukaryotes. Genomics 1998;54:443-452.

25. Hoki Y, Araki R, Fujimori A, Ohhata T et al. Growth retardation and skin abnormalities of the RECQL4-deficient mouse. Hum Mol Genet 2003;12:2293-2299.

26. Rothmund A. Uber cataracten in verbindung mit einer eigentumlichen hautdegeneration. Arch Klin Exp Ophtal 1868;4:159-182.

27. Thomson MS. Poikiloderma congenitale. Br J Dermatol 1936;4:221-234.

28. Taylor WB. Rothmund's syndrome-Thomson's syndrome. Arch Dermatol 1957;75: 236-244.

29. Wang LL, Gannavarapu A, Kozinetz CA, Levy ML et al. Association between osteosarcoma and deleterious mutations in the RECQL4 gene in Rothmund-Thomson syndrome. J Natl Cancer Inst 2003;95:669-674.

30. Wang LL, Levy ML, Lewis RA, Chintagumpala MM et al. Clinical manifestations in a cohort of 41 Rothmund-Thomson syndrome patients. Am J Med Genet 2001;102:11-17.

31. Piquero-Casals J, Okubo AY, Nico MM. Rothmund-thomson syndrome in three siblings and development of cutaneous squamous cell carcinoma. Pediatr Dermato 2002;19:312-316

32. Pianigiani E, De Aloe G, Andreassi A, Rubegni P, Fimiani M. Rothmund-Thomson syndrome (Thomson-type) and myelodysplasia. Pediatr Dermatol 2001;18:422-425.

33. Kitao S, Shimamoto A, Goto M, Miller RW et al. Mutations in RECQL4 cause subset of cases of Rothmund-Thomson syndrome. Nat Genet 1999;22:82-84.

34. Kaariainen H, Ryoppy S, Norio R. RAPADILINO syndrome with radial and patellar aplasia/hypoplasia as main manifestations. Am J Med Genet 1989;33:346-351.

35. Kant SG, Baraitser M, Milla PJ, Winter RM. Rapadilino syndrome-a non-Finnish case. Clin Dysmorphol 1998;7:135-138.

36. Hilhorst-Hofstee Y, Shah N, Atherton D, Harper JI et al. Radial aplasia, poikiloderma and auto-immune enterocolitis-new syndrome or severe form of Rothmund-Thomson syndrome? Clin Dysmorphol 2000;9:79-85.

37. Kellermayer R, Siitonen HA, Hadzsiev K, Kestila M, Kosztolanyi G. A patient with Rothmund-Thomson syndrome and all features of RAPADILINO. Arch Dermatol 2005;141:617-620.

38. Baller F. Radiusaplasie und Inzucht. Z Menschl Vererb 1950;29:782-790.

39. Gerold M. [Healing of a fracture in an unusual case of congenital anomaly of the upper extremities]. Zentralbl Chir 1959;84:834.

40. Seto ML, Lee SJ, Sze RW, Cunningham ML. Another TWIST on Baller-Gerold syndrome. Am J Med Genet 2001;104:323-330.

41. Gripp KW, Stolle CA, McDonald-McGinn DM, Markowitz RI et al. Phenotype of the fibroblast growth factor receptor 2 Ser351Cys mutation: Pfeiffer syndrome type III. Am J Med Genet 1998;78:356-360. 